\title{
An elusive brain death diagnosis You can't get there from here
}

\author{
Daryl Story, MD \\ Stephen M. Winter, MD
}

here are approximately 100,000 patients in the United States waiting for donated organs. Annual mortality due to insufficient organ availability is considerable. An organ donor must satisfy the "dead donor rule": removal of organs itself cannot result in death. Therefore it is of utmost importance to be sure that a potential donor is truly brain dead. Uncertainty or delay in diagnosis reduces the availability of transplantable organs.

\section{Case report}

A 69-year-old man with bipolar disorder and hypercholesterolemia was found unresponsive. Seizures were described by a witness. The patient was intubated in the field by emergency medical services and given diazepam $10 \mathrm{mg}$ IV for recurrent seizures. Blood pressure was 220/110 mm Hg. In the emergency department, examination revealed coma, extensor posturing, and breath initiation, but no other brainstem reflexes. Medications included quetiapine, lamotrigine, pregabalin, and clonazepam $0.25 \mathrm{mg}$ qhs. CT scan of the brain showed a large intracerebral hemorrhage (figure 1A). Laboratory results were unremarkable.

The patient deteriorated. Examination 5 hours into hospitalization revealed blood pressure $140 / 96 \mathrm{~mm} \mathrm{Hg}$ and normothermia. There was no eye opening to noxious stimulus. Pupils were $6 \mathrm{~mm}$ and fixed, oculocephalic reflex was absent, corneal reflexes were absent bilaterally, cold calorics were absent, and there was no motor response to central or peripheral pain. The apnea test, following American Academy of Neurology guidelines, ${ }^{1}$ was positive, with no respiratory effort despite $\mathrm{CO}_{2}$ elevation above $60 \mathrm{~mm} \mathrm{Hg}$.

Ancillary testing was requested due to presence of factors potentially confounding clinical assessment. The patient had received sedating medications including clonazepam daily and diazepam had been recently given.

A CT angiogram (CTA) showed evidence of cortical perfusion by opacification of middle cerebral branches (figure 1B). Brain death could not be confirmed based on this result. The family elected for continued support in hopes that progression would allow the desired organ donation to go forward. On day 2, repeat CTA showed similar findings (figure 1C). EEG on that day showed cortical electrical activity (figure 2).

On the evening of day 2 , the patient experienced a cardiac arrest and could not be resuscitated.

Stroke Center (DS) and Pulmonary and Critical Care Medicine (SMW), Norwalk Hospital, CT; New York Medical College (DS), Valhalla; and Yale University (SMW), New Haven, CT.

Funding information and disclosures are provided at the end of the article. Full disclosure form information provided by the authors is available with the full text of this article at Neurology.org/cp.

Correspondence to: Daryl.Story@Norwalkhealth.org 
Figure $1 \quad \mathrm{CT}$ and $\mathrm{CT}$ angiogram findings
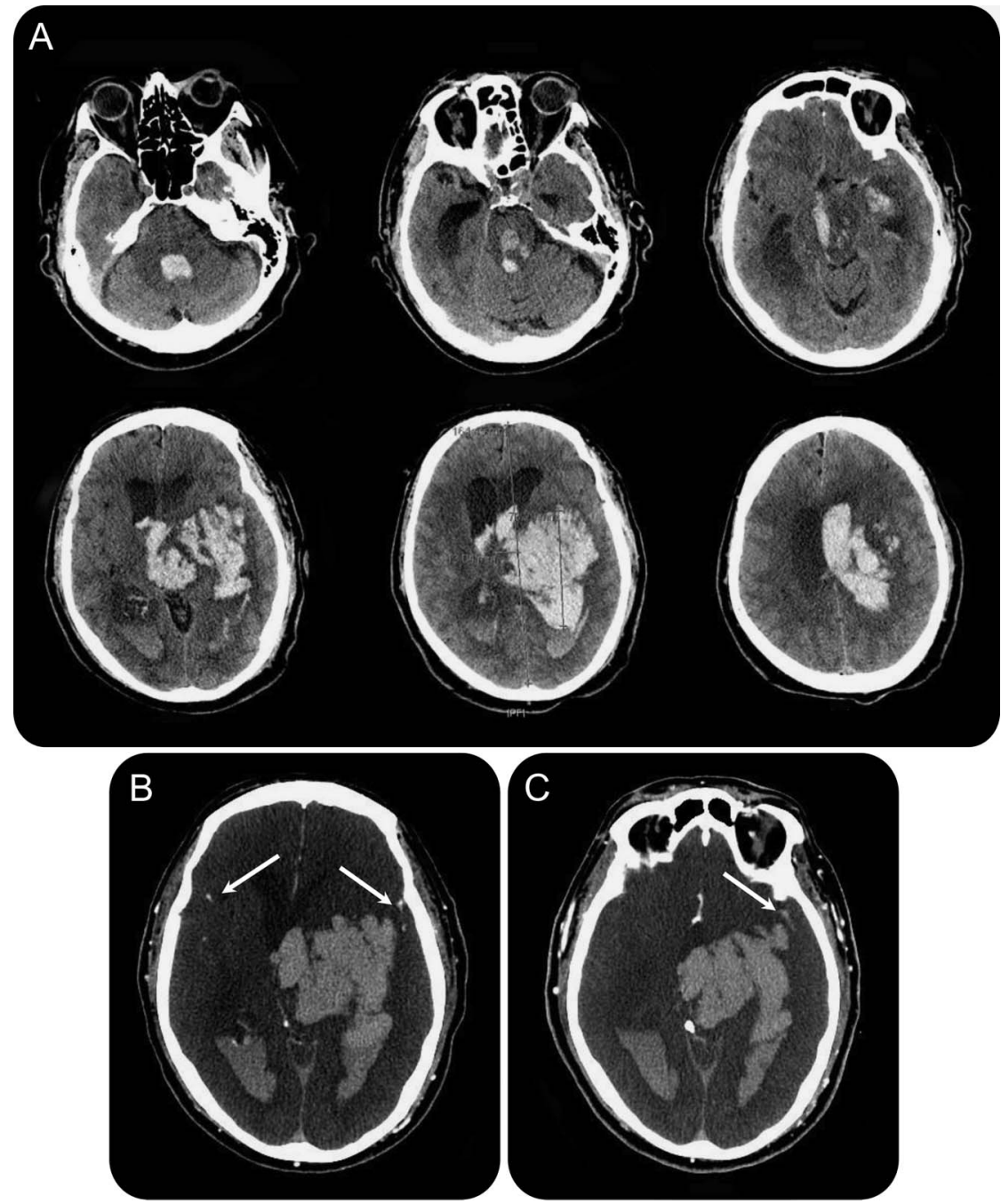

(A) Initial CT scan shows large intracerebral and intraventricular hemorrhage. (B) Day 1 CT angiogram (CTA) shows evidence of bilateral arterial blood flow in middle cerebral artery (MCA) branches. (C) Day 2 CTA shows continued evidence of arterial blood flow in left MCA branch.

\section{DISCUSSION}

It can be questioned whether ancillary testing was necessary in this patient with a clinical examination consistent with brain death and probably low levels of sedating medications. However, no timely laboratory levels were available.

Another question regards the use of CTA as an ancillary test. The American Academy of Neurology does not endorse its use due to insufficient evidence ${ }^{1}$ in spite of acceptance outside the United States. ${ }^{2,3}$ We believed that a positive CTA would be helpful since clinical certainty of brain death by examination was high. We used a protocol associated with sensitivity of $85.7 \%$ and specificity of $100 \%$ for the diagnosis of brain death. ${ }^{2}$ EEG was notably in agreement with the CTA findings.

A final question involves current practice and the concept of brain death for the purposes of organ procurement. Our patient was unconscious and had a hopeless prognosis. By our following protocol, best clinical judgment, and the dead donor rule, he could not fulfill a wish to donate organs.

This issue has been addressed in the literature. Wijdicks ${ }^{4}$ suggests foregoing ancillary testing, which can be confusing, and proceeding to a donation after cardiac death protocol when 
Figure 2 Electroencephalogram findings



Day 2 EEG shows presence of brain wave activity.

there is uncertainty about brain death by clinical examination. Truog and Miller ${ }^{5}$ argue that the means of diagnosing brain death are flawed, and organs may in some cases be taken when the diagnosis is false. Our patient validates this point: either the clinical examination or the ancillary testing was incorrect. The authors go on to suggest foregoing the dead donor rule, and allowing organ procurement with devastating, irreversible neurologic injury as the chief criterion, with informed consent. Veatch ${ }^{6}$ suggests redefining brain death as the total loss of brain functions responsible for consciousness, a "higher brain" definition of death.

Pathologic studies of brain tissue 12-36 hours after brain death diagnosis show wideranging degrees of spared neurons, and seldom a diffusely necrotic "respirator brain" appearance. $^{7}$ These regions of spared cells may produce electrical activity and receive blood flow, even in a patient diagnosed as brain dead clinically. We believe this situation is what led to our CTA and EEG findings. Our patient likely had cortical metabolic activity, though was rendered permanently unconscious by severe brainstem injury. He was in the border zone between life and death. Science, religion, ethics, and societal priorities all have a stake in how best to handle such a complicated and important issue.

\section{REFERENCES}

1. Wijdicks EF, Varelas PN, Gronseth GS, Greer DM; American Academy of Neurology. Evidence-based guideline update: determining brain death in adults: report of the Quality Standards Subcommittee of the American Academy of Neurology. Neurology 2010;74:1911-1918.

2. Frampas E, Videcoq M, de Kerviler E, et al. CT angiography for brain death diagnosis. AJNR Am J Neuroradiol 2009;30:1566-1570.

3. Shemie SD, Lee D, Sharpe M, Tampieri D, Young B; Canadian Critical Care Society. Brain blood flow in the neurological determination of death: Canadian expert report. Can J Neurol Sci 2008;35: $140-145$.

4. Wijdicks EF. The case against confirmatory tests for determining brain death in adults. Neurology 2010;75:77-83.

5. Truog RD, Miller FG. The dead donor rule and organ transplantation. N Engl J Med 2008;359: 674-675.

6. Veatch RM. Donating hearts after cardiac death: reversing the irreversible. N Engl J Med 2008;359: 672-673.

7. Wijdicks EF, Pfeifer EA. Neuropathology of brain death in the modern transplant era. Neurology 2008;70:1234-1237. 


\section{STUDY FUNDING}

No targeted funding reported.

\section{DISCLOSURES}

The authors report no disclosures. Full disclosure form information provided by the authors is available with the full text of this article at Neurology.org/cp.

\section{Share Your Knowledge}

Neurology ${ }^{\circledR}$ Clinical Practice encourages readers to share their insights, expertise, and experiences.

- How are you employing drugs and devices in your field?

- What ethical challenges do you face?

- Do you have a case report that is illustrative of a clinical challenge?

- What challenges have you faced or successes have you enjoyed in bringing greater efficiency to your practice?

If you are interested in delivering a high-quality, peer-reviewed message to your colleagues in practice, submit your paper online. 